\title{
Implementación de instrumentos básicos para un laboratorio con tecnología RFID
}

\section{RESUMEN}

Con la implementación de los equipos básicos de la tecnología de identificación por radiofrecuencia (RFID)1', se capacitará en mejores condiciones a los docentes de la especialidad de Ingeniería Industrial y de Ingeniería Textil y confecciones, asi como a sus estudiantes de pre y posgrado de la facultad de Ingeniería Industrial. Con esta nueva y moderna tecnología el laboratorio se ubica entre las mejores y modernas instalaciones de las universidades públicas y privadas. Al introducir en la currícula el laboratorio de tecnología de identificación por radiofrecuencia (RFID), se podrá capacitar a los estudiantes y se difundirá esta capacitación hacia los trabajadores de empresas, buscando mejorar la enseñanza-aprendizaje sobre: la reducción de inventarios, trazabilidad de pedidos, reducción de costos operativos y laborales, mejor uso de los activos reutilizables de la empresa (pelets, carretillas de carga, etc.).

Palabras claves: reducción de inventarios, identificación de mercaderías, reutilizables de la empresa.

DESIGN OF A MODEL OF MANAGEMENT FOR THE DEVELOPMENT OF THE SMALL AGRICULTURAL RURAL UNITS OF PERU ASOCIATIVIDAD'S WORKSHOPS

\section{ABSTRACT}

With the implementation of the basic equipment of radio frequency identification technology (RFID) 1 , are better able to train teachers in the specialty of Industrial Engineering and Engineering Textiles and clothing, and their undergraduate and graduate Industrial Engineering faculty. With this new, modern laboratory technology is among the best and modern facilities of public and private universities. By introducing the curriculum laboratory identification technology (RFID), may enable students and disseminate this training to the employees of companies, seeking to improve the teaching-learning: reducing inventories, tracking of orders, reducing operating costs and labor, better use of reusable assets of the company (pellets, cargo trucks, etc.).

Keywords: Inventory reduction, identification of goods, reusable business

\section{INTRODUCCIÓN}

La presente investigación en su primera etapa trata de implementar un centro de operaciones denominado "Laboratorio de identificación por radio frecuencia (RFID)* en el conocimiento que es una de las tecnologías nuevas en nuestro medio más prometedoras que se han orientado al sector del almacenamiento y distribución en muchos años.

Uno de los objetivos de mediano plazo es comprobar el beneficio que traería a la Facultad tanto en enseñanza como en aprendizaje de nuevas técnicas de almacenamiento y distribución de mercaderías.

El iniciar una prueba piloto cuando todo el equipamiento del laboratorio este completo servirá para investigar la operatividad, costos y utilidad en las empresas del Perú.

El adaptar la tecnología del RFID a nuestra realidad empresarial, es uno de los objetivos de mediano plazo, puesto que con este trabajo lo que se pretende es identificar las empresas localizadas en el Perú que ofrezcan con ventajas para la universidad la adquisición e implementación de un laboratorio RFID y que se comprometa con la capacitación para la difusión de la importancia de esta tecnología.

Se espera que con la implementación del laboratorio y las adquisiciones progresivas que complementen al laboratorio básico del sistema RFID, proporcionen mayores ventajas y permitan mejorar la curva de aprendizaje de los estudiantes que pretendan incursionar en proyectos a gran escala dentro del país y de las empresas en general.

El sistema de identificación por radio frecuencia, trata de reducir costos, minimizar tiempos de espera, agilizar procesos y mayor control de una organización, que son parte de las enseñanzas de la Ingeniería Industrial y de textiles que se complementarán con eficiencia en el curso de logística y aprenderán los estudiantes algo novedoso para nuestro medio.

El objetivo general es demostrar en la práctica la factibilidad de la instalación a nivel básico de un laboratorio RFID, que permita evaluar las ventajas, costos y sobretodo el aprendizaje de nue-

1 Dra. en Ingeniería Industrial, Profesor Principal de la Facultad de Ingeniería Industrial de la UNMSM, teogaza57@yahoo.es

2 Ingeniero Industrial de la PUCP administrador Decor Instala, jqg@hotmail.com.

* RFID: Radio Frequency Identification (Identificación por radio frecuencia). 
vos conocimientos de automatización en el manejo de inventarios. Como objetivos específicos se encuentran: a) adquirir e instalar la instrumentación básica de un laboratorio RFID, b) capacitar a los docentes y estudiantes en el uso del laboratorio y c) Capacitar al personal encargado de su mantenimiento y a trabajadores de empresas que deseen incursionar en esta nueva tecnología.

\section{MARCO TEÓRICO Y PLANTEAMIENTO DE HI- PÓTESIS}

\section{MARCO TEÓRICO}

\subsection{Antecedentes del sistema RFID}

En el año 2003, el líder del retail mundial, el grupo estadounidense Wal-Mart solicitó a sus proveedores que colocaran a sus productos etiquetas con tecnología de identificación por radiofrecuencia. También lo pidieron las compañías alemanas, Metro e inglesa, Tesco. Los fabricantes decidieron también dar el paso, así Nestlé, Henkel, Procter \& Gamble o Gillette, quiénes cuenta ya con más de 500 millones de chips implantados en sus productos como experimentación (Noticiasdot. com, 2003: [1]).

Desde el 2008, la adopción de las tecnologías de identificación por radiofrecuencia se está generalizando en las empresas de distribución y logística en España. Así lo expone el estudio "Estado actual de RFID en España" elaborado por la Asociación Europea para la Identificación Segura, ID Track, en colaboración con IBM (Gomis, 2008, [2]).

"Del citado informe se puede extraer la conclusión de que un $30 \%$ de las empresas consultadas ha implantado ya tecnologías RFID y el $83 \%$ se muestra satisfecho con los resultados obtenidos. Sin embargo, esta tecnología no es totalmente conocida por las compañías, ya que un $28 \%$ aseguró no conocerla, aunque, entre aquellos que sí la conocen, el 54\% apunta a la gestión de materiales como la aplicación más valorada, seguida de la gestión de stocks $(33,3 \%)$, la logística $(26,4 \%)$ o la automatización industrial $(18,1 \%)$ (Perú retail 2006, [3]). Entre las principales ventajas que aporta RFID para las empresas encuestadas, destaca la posibilidad de disponer de un mayor control en todo el proceso de distribución de los productos $(58,1 \%)$, seguido de la reducción de errores en la información de productos $(32,3 \%)$ y de la identificación de forma individual de cada unidad $(25,8 \%)$ (Ídem, [3]).
Por último, entre las razones que encuentran las organizaciones para no implantar un sistema de RFID se encuentra la falta de necesidad, con un 42,9\%; el alto coste, con un $12,9 \%$ y la falta de información, con un $10 \%$ ". En base a estas cifras, Luís Soler Gómez, director de IDTrack, manifiesta su convencimiento de que la RFID "es una tecnología de futuro" (Ídem, [3]).

De igual forma piensa Labarga Manuel O., director de tecnologías emergentes de IBM España, Portugal, Grecia, Israel y Turquía, quien destaca cuatro claves para que una implantación RFID tenga éxito: que este basada en estándares, es decir, en entornos abiertos ya que ha de ser escalable y fiable; que se de una mayor visibilidad de las ventajas que puede aportar a otras áreas; que se produzca un estudio en el que se analicen claramente los beneficios de negocio y que sea una área de innovación dentro de la empresa. (Ídem [3])

Si bien es cierto que cuando una tecnología emerge los costos son altos y en la medida del uso que se les de, el mayor conocimiento y difusión de sus ventajas, la satisfacción de necesidades y mayor aplicabilidad, estos tienden a ser más asequibles al usuario. Justamente en la medida en que el precio de las etiquetas para este sistema bajo los departamentos logísticos de las empresas están prestando mayor atención a la posibilidad de integrar esta tecnología en los procesos de negocio, con el objetivo de mejorar la visibilidad y la precisión de las operaciones logísticas (Egoméxico, [4]).

El avance de la tecnología en el entorno de esta gran herramienta para la industria estimula a los usuarios o ciudadanos que de una forma u otra se vean involucrados en la aplicación de una tecnología, lo cual si bien favorece los procesos, pueden afectar a la sociedad en forma de seguridad y derechos de los ciudadanos. El debate surgió hace años al tiempo que comenzaba a gestarse la tecnología de identificación por radiofrecuencia. A medida que avanza, avanzan también los temores.

Para IDTrack, buena parte de estas empresas dedican esta tecnología a los procesos de almacén, logística y el centro de distribución. Mientras que, su implantación "está ligada al tipo de procesos que están haciendo las empresas, como la gestión del inventario, el control de la logística, la eficiencia en la distribución, la prevención de pérdidas y el time-to-market". 
Asimismo, la adopción de esta tecnología se estará iniciando en forma progresiva, en el Centro de Manufactura Avanzado (CEMA) de Industriales para pasar luego a las empresas evaluando su cadena de producción y finalmente abarcar la cadena de valor que incluye al cliente.

\subsection{EMPRESAS CONSIDERADAS COMO LAS MÁS CONFIABLES Y DE MÁXIMA SEGURIDAD EN EL MUNDO}

\section{a. Empresas internacionales:}

LENEL ONGUARD, es considerado el mejor sistema integrado de seguridad en el mundo.

RBH CONTROL DE ACCESO, los sistemas de Control de Acceso RBH Integra 32 y Axiom V son profesionales y muy confiables.

PELCO ENDURA, El sistema Endura de Pelco es el plataforma del futuro de IPTV de Pelco.

b. Empresas ubicadas en el Perú

\section{GS1 PERÚ}

Presenta el primer centro de competencias y laboratorio de uso de la tecnología de la identificación por radio frecuencia (RFID) y código electrónico de productos (EPC). (5)

\section{DMS-PERÚ}

DMS Perú es una empresa líder en el desarrollo e integración de soluciones para la captura automática, impresión especializada, soluciones móviles y redes inalámbricas, con productos, sistemas y soluciones de alto desempeño y de alta calidad comprobada.

1.3. Que se entiende por RFID

Figura N. ${ }^{\circ}$ 1: Confección de RFID

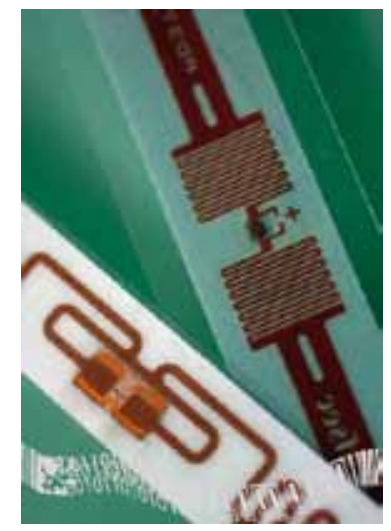

RFID (Radio Frecuency Identification) es un sistema de identificación en la que a través de un chip, un elemento cualquiera puede tener una identificación única (ver Fig.1 y 2).

Este sistema hace uso de señales de radiofrecuencia, similar a las señales de radio, redes wireless, celulares, etc (ver Fig. 3).

EI RFID es en esencia un identificador basado en radiofrecuencia. Se define como una herramienta tecnológica de identificación cuya principal premisa es sustituir al código de barras actualmente existente. Dicha tecnología ofrece un sistema único de localización en tiempo real a distancia que permite monitorizar cualquier parámetro referente al objeto que la comporte (Llamazares, 2009, [6]).

Se trata de una tecnología basada en la utilización de un pequeño chip adherido a un producto, y a través del cual es posible mantener un rastreo de su localización. La distancia de rastreo varía mucho, dependiendo del tamaño, tipo y antena del chip, pero podría ser desde $2 \mathrm{~cm}$ a $13 \mathrm{~m}$ sencillos, hasta incluso varios kilómetros en los más complejos. Son realmente pequeños y tal y como van los avances, en poco tiempo podrían ser considerados virtualmente invisibles. (Egomexico, [1])

Figura N. ${ }^{\circ}$ 2: Elemento componentes del sistema RFID

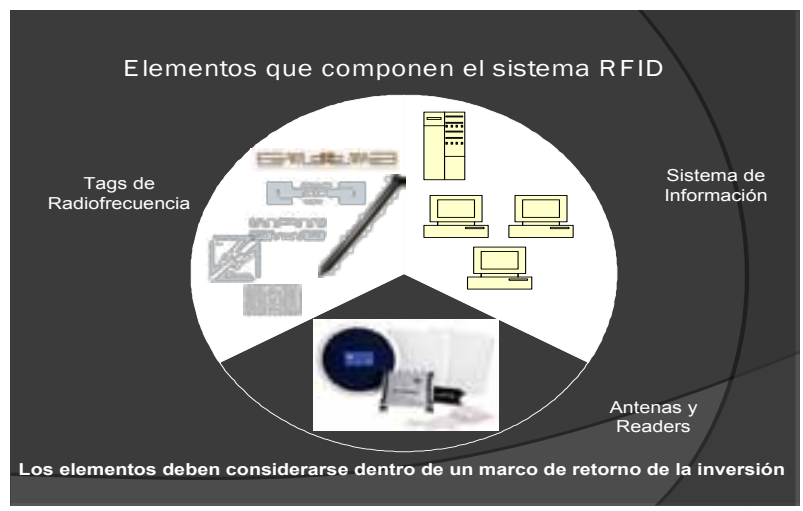

Figura $N^{\circ}$ 3: Transferencia de señales por radio frecuencia

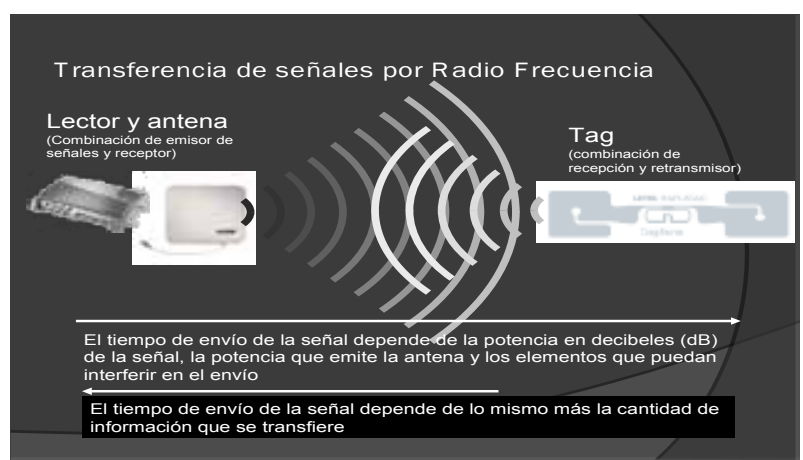




\section{VENTAJAS DEL SISTEMA}

Algunos aspectos a destacar son: (ONTSI, [7])

- Reducción de inventarios como resultado de una mejor visibilidad del stock. Permite llevar a cabo la trazabilidad y visibilidad a lo largo de la cadena de producción y de suministro, con mayor fiabilidad y agilidad que con los sistemas actuales, lo que ayuda a las empresas a adoptar políticas de planificación de stocks más ajustadas a las necesidades reales.

- Mejora del nivel de servicios. Gracias a un mayor control en las entregas de los pedidos a clientes, se puede asegurar una trazabilidad de pedidos mucho más fiable, lo que se traduce en una disminución de errores y por tanto devoluciones, redundando en ahorro de costes administrativos y en aumento de ventas.

- Mejora de la eficiencia y reducción de costes operativos y laborales. Los procesos de gestión logística en almacenes se simplificarán enormemente al no ser necesarias las operaciones de escaneo para identificar los productos en cada una de las fases por donde pasan (producción, almacén de producto terminado, centro de distribución, picking, expedición, recepción en el punto de venta, etc.).

- Exactitud en la identificación de mercancía. Como la recogida de información no depende de operaciones manuales, se consigue aumentar notablemente la seguridad y exactitud en el proceso de altas y bajas de inventarios.

- Disminución de la pérdida por hurto externo, interno o errores administrativos, como consecuencia de las mejoras mencionadas anteriormente.

- Mejor uso de los activos reutilizables de la empresa (embalajes reutilizables, paletas, carretillas de carga, etc.).

- Lucha contra la falsificación de productos. La identificación unívoca de productos y la accesibilidad de esos datos a través de Internet hace mucho más fácil el control del producto comercializado.
En general, si continuamos en el perfeccionamiento de este laboratorio básico, la identificación por medio de radiofrecuencia (RFID) aportará una notable mejora en los mecanismos de seguimiento de los envíos a través de las cadenas globales de distribución si se trata de exportaciones de las empresas, permitiendo así que tanto proveedores como distribuidores y clientes, puedan disponer de información real del número de productos y de las fechas de entrega.

Permite además detectar las fases del proceso en las que se produce un alto índice de pérdida desconocida, ralentizaciones, etc, con el objetivo de aliviar cuellos de botella y reducir de forma considerable la falta de productos en las tiendas.

\section{APLICACIONES}

Son muchas las empresas y organizaciones que pueden beneficiarse de las ventajas de la tecnología RFID. Algunas de sus aplicaciones son las siguientes: la localización y el seguimiento de objetos.

\section{Control de accesos \\ Identificación de materiales \\ Control de fechas de caducidad}

Almacenaje de datos

Control de stocks

Automatización de los procesos de fabricación

Reducción de tiempo y coste de fabricación

Reducción de colas a cancelar en caja

Inventario automático

Entre las aplicaciones con las que se iniciaría el laboratorio sería el etiquetado RFID de los productos finales bien a nivel de ítem, caja o palé de una determinada empresa a tomarla como piloto, esto permitirá abordar de forma más eficiente la gestión de expediciones, automatizando los procesos asociados a la salida de productos y controlando que cada pedido sea servido con los elementos correctos, hacer este seguimiento es materia del trabajo de investigación propiamente dicho, por que luego de probar el funcionamiento nos permitirá calcular costos de implementación y verificar el beneficio costo para la empresa.

Los productos (ítem, caja o palé) seleccionados dentro de la empresa serían etiquetados al final del proceso de producción con etiquetas de RFID que contienen la información suministrada por el sistema de información de la empresa. Una vez que los 
productos pasan a la zona de carga, se puede verificar de forma automática que corresponden con la orden de expedición adecuada, todo ello con una reducción sustancial de la intervención humana en este proceso de comprobación, pudiendo además generar las instrucciones adecuadas para el personal de expediciones en caso de necesidad de intervención, con el objetivo principal de evitar que las expediciones con errores salgan de las instalaciones, lo cual reduce drásticamente los costes asociados a la reparación de los mismos. Si estas pruebas se pueden realizar con los equipos básicos que se están adquiriendo sería de gran estímulo para nuestro trabajo, caso contrario debemos complementar con aquellos equipos que aún se requieran.

Otra de las aplicaciones que se puede iniciar sería la de gestión de almacén inteligente, la automatización de la gestión de almacén mediante el uso de la tecnología RFID implica una mejora substancial en todo el proceso fundamentada en la reducción de los tiempos de inventariado y la optimización de los stocks, lo que facilita las decisiones de producción adecuándolas a las necesidades reales. De esta forma se puede conseguir un importante ahorro de espacio de almacenaje.

Los diversos especialistas e investigadores señalan que esta es una tecnología del futuro que hará cambiar nuestras vidas en todos los campos de las actividades, sociales, culturales, políticas, de vigilancia, en el campo de la salud, etc. Por lo que el laboratorio a implementarse representaría un inicio de lo que sería en realidad un laboratorio RFID de los que ya existen, pero que sí es válida como iniciativa para fomentar el interés de los estudiantes y empresarios.

Si bien esta tecnología la han diseñado principalmente para facilitar el trabajo de comercialización de productos de consumo en diferentes áreas, han encontrado otros usos a la posibilidad de seguir la vida de un chip durante un determinado periodo de tiempo. Proponen algunos ejemplos que se toman para explicar mejor lo que se espera en un futuro próximo para el país; para el control de las entradas a espectáculos, para el control de acceso a las carreteras de peaje. Por ejemplo, en los hospitales, un brazalete puesto a un paciente, lo identifica y asocia en cualquier lugar y momento con la medicación y régimen alimenticio que deba seguir.

La comunidad europea piensa introducir esta técnica en la próxima generación de billetes para entre otras cosas facilitar el recuento de billetes. En bibliotecas y centros de documentación se puedan localizar rapidamente los libros y documentos solicitados, etc.

\section{FORMULACIÓN DEL PROBLEMA:}

¿La implementación del laboratorio básico con tecnología RFID conseguirá mantener a la facultad a la vanguardia del conocimiento en esta materia, a través de la capacitación de sus estudiantes de pre y posgrado y a trabajadores de las empresas?

\section{PLANTEAMIENTO DE HIPÓTESIS}

La hipótesis del trabajo de investigación consiste en:

Demostrar si la aplicación tecnológica de identificación por radio frecuencia (RFID) que se instalará en el Centro de Manufactura Avanzada (CEMA) puede tener resultados exitosos en la enseñanza aprendizaje de los estudiantes de la facultad y de las empresas del país que pongan a la vanguardia de este conocimiento a la facultad.

\section{MÉTODOS Y RESULTADOS}

\subsection{Método}

El desarrollo de la presente investigación trata sobre la implementación de un laboratorio con tecnología RFID, que permita introducir nuevos conocimientos en materia de identificación de bienes automatizadamente para lo cual se desarrollará tres fases:

a. La primera fase tiene como contenido una explicación precisa que permita la comprensión sobre el funcionamiento de la tecnología de identificación por radio frecuencia (RFID), teniendo en cuenta las diversas opiniones de empresarios y expertos en el tema. Es así que la investigación se ha centrado en esta etapa, se ha capacitado a profesores y estudiantes en esta Tecnología RFID asistiendo a charlas y conferencias que presentaban los expertos de la empresa GS1-EPC-Global, ubicada en Lima, Perú.

b. La segunda fase corresponderá al estudio y análisis de la aplicación de la tecnología RFID instalada en la facultad, que permita identificar las ventajas y costos en que se incurriría para la instalación de esta tecnología en las empresas. Así como identificar el potencial interés de los estudiantes en nuevas tecnologías para el aprendizaje y capacitación orientada a competencias.

c. La tercera fase se estudiará la viabilidad de complementar la tecnología con nuevos equipos para ir incursionando en otras actividades que nos permita esta tecnología y que se oriente a la mejora de la gestión de la facultad y de la universidad misma, así como trabajos coordinados con empresas. 
Continuando con el método que se empleó, se menciona que para desarrollar la primera fase se tuvo que recolectar información bibliográfica relacionada con el tema, publicaciones de reportajes en revistas, Internet, visitas que realizamos en España durante una breve permanencia a personas que tiene relación con el tema, visitas a empresas que ya están aplicando la tecnología, entrevistas con los empresarios que usan la tecnología y charlas y conferencias recibidas en la ciudad de Lima.

Luego, se analizó esta información y se estructuró de tal manera que el lector pueda comprender los aspectos innovadores sobre la aplicación de esta tecnología.

Una vez que se analice la implementación del laboratorio se procederá con las siguiente dos fases con el fin de plasmar los potenciales beneficios que aportaría a la facultad y a las empresas la aplicación de la tecnología estudiada con el objetivo de que a corto o medio plazo se consideren los aportes una realidad que permitirá continuar profundizándose en el tiempo para bienestar de nuestros estudiantes y de la comunidad empresarial.

A continuación se presentan las etapas del estudio:

1. Diseño de planta del laboratorio RFID (ver Figura N. $\left.{ }^{\circ} 5\right)$.

2. Ejecución de obras adicionales para acondicionar el laboratorio RFID.

3. Adquisición e instalación de la instrumentación básica.

4. Pruebas preliminares.

5. Capacitación.

\section{DISTRIBUCIÓN FÍSICA DE LOS EQUIPOS ADQUIRIDOS Y LAS SALAS DE CAPACITACIÓN ASÍ COMO OFICINAS}

Figura № 5: Distribución física de los equipos

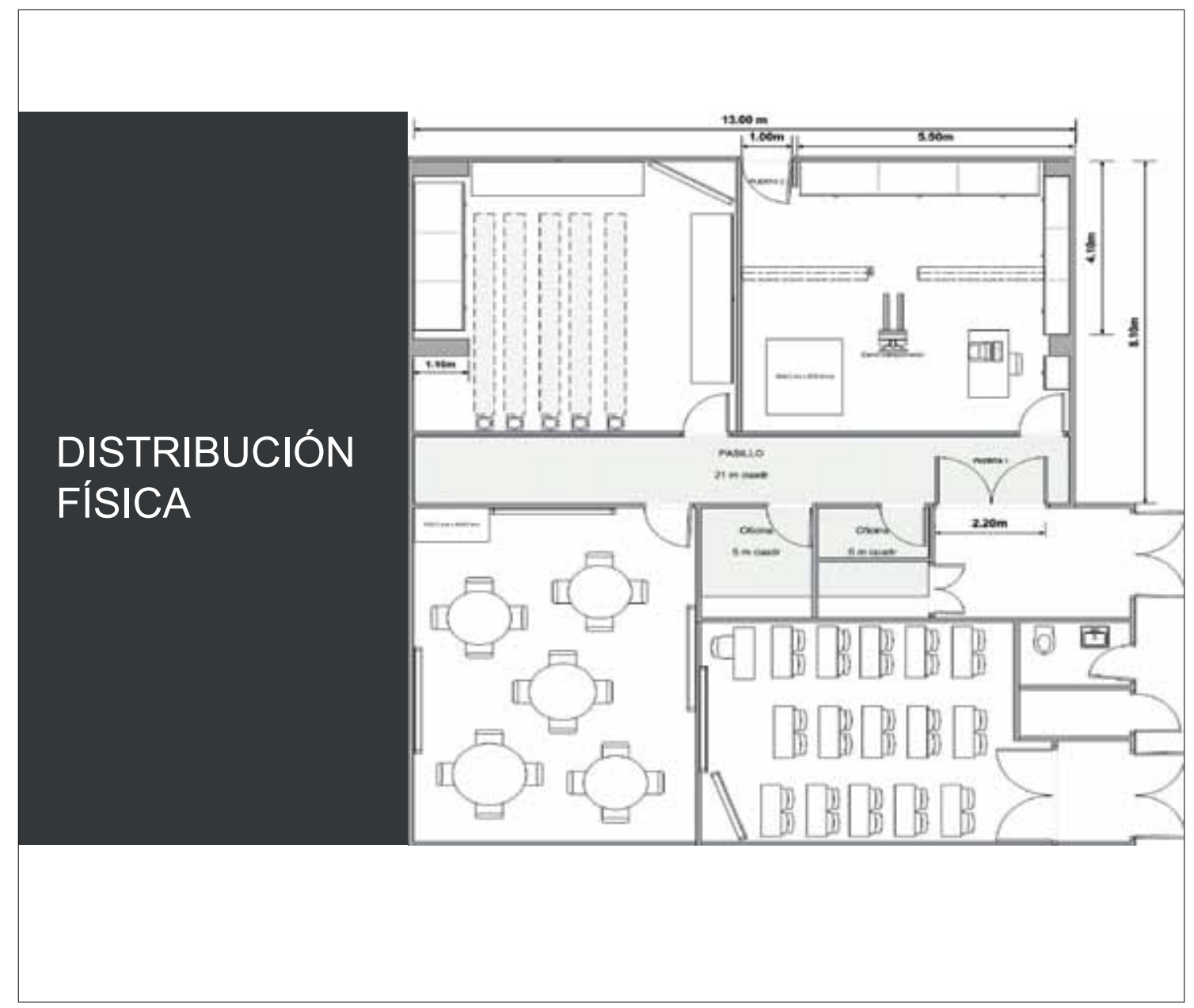

Fuente: elaboración del equipo de trabajo del estudio 
Complementariamente, se requiere de lo siguiente:

1. Instalación de un arco para el proyecto (Ver Fig.6).

2. Instalación de 6 antenas.

3. Instalación de un transportador.

4. Realización de dos sesiones de capacitación para docentes y personal del laboratorio respectivamente.

Figura $N .^{\circ} 6$ : arco para la recepción de la señal

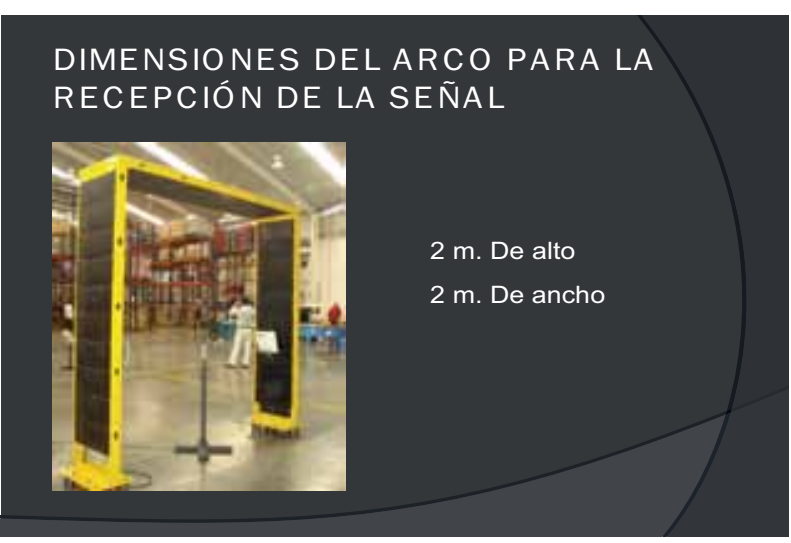

Fuente: Fuente: Llamazares, Juan Carlos

Los gastos que irroguen estas actividades complementarias estará a cargo de la facultad quien contribuirá para que se haga realidad esta nueva tecnología, está aprobado por el Consejo de Facultad se destine en principio $8,000.00$ nuevos soles, a la fecha el presupuesto total asciende a S/. 26,997.28 nuevos soles
PRESUPUESTO:

\begin{tabular}{|c|c|c|c|}
\hline Cant. & Descripción & $\begin{array}{l}\text { Precio } \\
\text { unitario } S / . \\
(\sin I G V)\end{array}$ & $\begin{array}{l}\text { Precio } \\
\text { total S/. (sin } \\
\text { IGV) }\end{array}$ \\
\hline 2 & $\begin{array}{l}\text { Lectores RFID Impinj } \\
\text { Revolution }\end{array}$ & $5,747.90$ & $11,495.79$ \\
\hline 2 & $\begin{array}{l}\text { Elementos de corriente } \\
\text { para reader (Opcional si } \\
\text { no se cuenta con PoE) }\end{array}$ & 270.59 & 541.18 \\
\hline 1 & $\begin{array}{l}\text { Licencias de Software de } \\
\text { control de dirección }\end{array}$ & $2,124.36$ & $2,124.36$ \\
\hline 2 & $\begin{array}{c}\text { Convenios de garantías por } \\
3 \text { años (incluye la actualiza- } \\
\text { ción del Firmware durante } \\
\text { ese periodo) }\end{array}$ & $2,045.68$ & $4,091.36$ \\
\hline 1000 & $\begin{array}{c}\text { Tags UPM Dog Bone } \\
\text { Monza3 }\end{array}$ & 0.68 & 676.47 \\
\hline \multirow[t]{3}{*}{6} & $\begin{array}{c}\text { Antenas Far field (polari- } \\
\text { zado Izq y Der) }\end{array}$ & 536.81 & $3,220.84$ \\
\hline & $\begin{array}{c}\text { Asesoría en integración } \\
\text { RFID al equipo de sistemas } \\
\text { de la UNMSM, traducidas en } \\
50 \mathrm{HH} / \text { Consultor especialista } \\
\text { en RFID }\end{array}$ & & $4,847.25$ \\
\hline & TOTAL & & $26,997.28$ \\
\hline
\end{tabular}

\section{Método de enseñanza en el laboratorio:}

La enseñanza comprenderá desde la parte de programación del software de control, conocimiento del Hardware y el manejo y funcionamiento de los equipos, finalmente el manejo de software a nivel de usuario y ejecución de cada módulo de aprendizaje que se proponga para el dictado del curso.

Resumen: Propuesta Total Modulo RFID

\begin{tabular}{|c|l|l|}
\hline Unidades & Descripción & $\begin{array}{l}\text { Precio Uni- } \\
\text { tario S/. }\end{array}$ \\
\hline 1 & Lectores RFID Impinj Revolution & 5747.90 \\
\hline 1 & Lectores RFID Impinj Revolution & 5747.90 \\
\hline 1 & Elementos de corriente para reader & 270.59 \\
\hline 1 & Elementos de corriente para reader & 270.59 \\
\hline 1 & Licencias de Software de control de dirección & 2124.36 \\
\hline 1 & Convenios de garantías por 3 años (incluye la actualización del Firmware durante ese periodo) & 2045.68 \\
\hline 1 & Convenios de garantías por 3 años (incluye la actualización del Firmware durante ese periodo) & 2045.68 \\
\hline 1000 & Tags UPM Dog Bone Monza3 & 676.47 \\
\hline 1 & Antenas Far field (polarizado Izq) & 536.81 \\
\hline 1 & Antenas Far field (polarizado Izq) & 536.81 \\
\hline 1 & Antenas Far field (polarizado Izq) & 536.81 \\
\hline 1 & Antenas Far field (polarizado Der) & 536.81 \\
\hline 1 & Antenas Far field (polarizado Der) & 536.81 \\
\hline 1 & Antenas Far field (polarizado Der) & 536.81 \\
\hline & Consultoría & 4847.25 \\
\hline & TOTAL & 26997.28 \\
\hline
\end{tabular}




\section{CARACTERISTICAS TÉCNICAS DE LOS EQUIPOS}

\section{Equipo de lectura RFID Speedway Revolution}

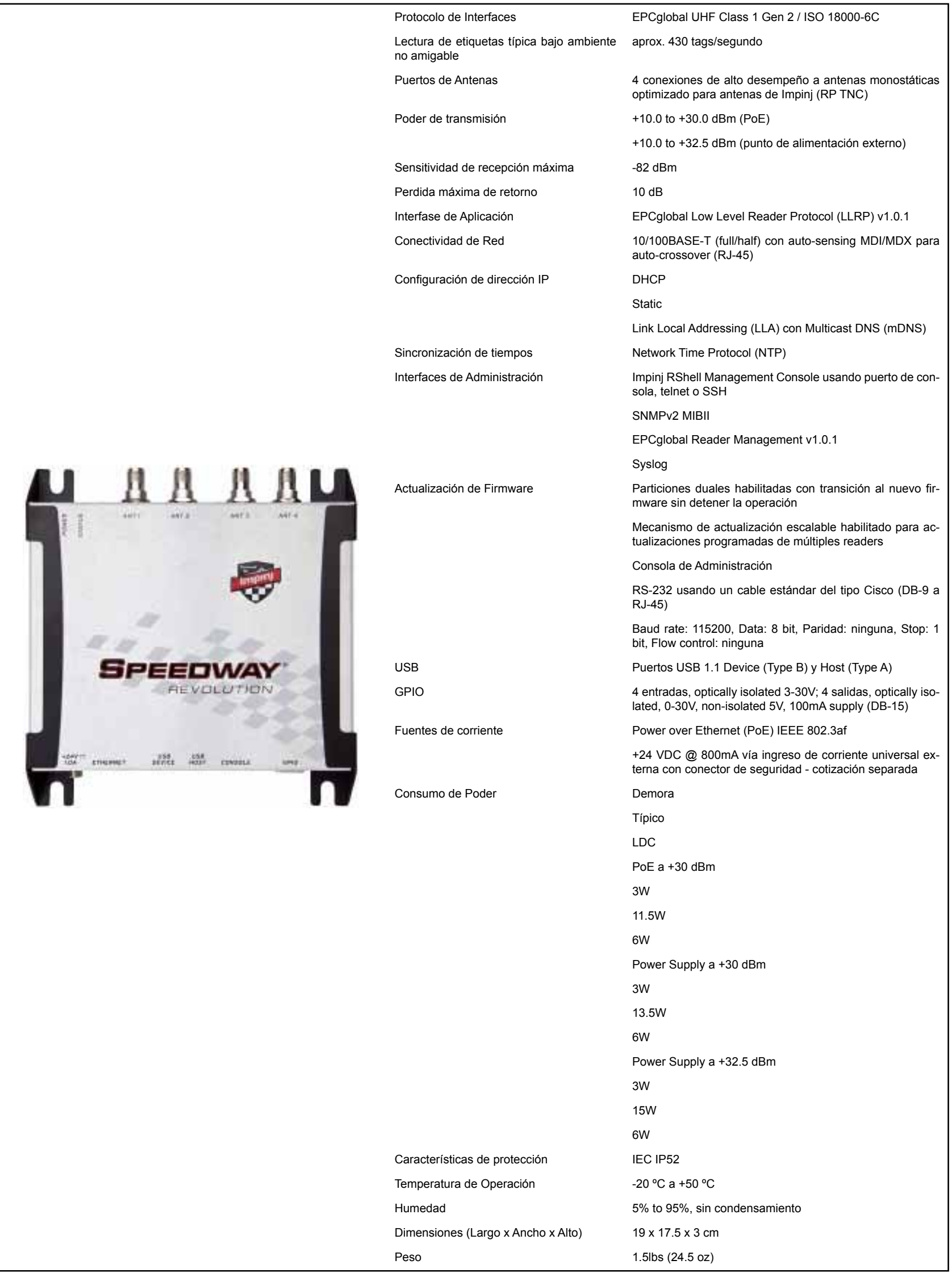


Elementos de conexión (Opcional si no se conecta a PoE)

\begin{tabular}{|l|l|}
\hline & $\begin{array}{l}\text { Conexión de abastecimiento de corriente universal con cable de } \\
\text { corriente alterna }\end{array}$ \\
\hline
\end{tabular}

\section{Etiqueta RFID - UPM DogBone}

\begin{tabular}{|lll|}
\hline & Tamaño de la antena & $93 \times 23 \mathrm{~mm}$ \\
& Tamaño del Die - cut (plastificado) & $97 \times 27 \mathrm{~mm}$ \\
& Ancho del rollo contenedor & $100 \mathrm{~mm}$ \\
& & Adhesivo permanente. -10 \\
& Temperatura del Adhesivo & ${ }^{\circ} \mathrm{C}$ a $120{ }^{\circ} \mathrm{C}$. \\
& Circuito Integrado (IC) & EPC Class 1 Gen $2-$ Monza3 \\
& Memoria EPC & 96 bits \\
& Frecuencia Operativa & $860-960 \mathrm{MHz}$ \\
& Retención de datos & 10,000 ciclos $/ 10$ años \\
& Temperatura de Operación & $-40{ }^{\circ} \mathrm{C}$ a $+85^{\circ} \mathrm{C}$ \\
& Diámetro posible para doblarlo (D) & $>50 \mathrm{~mm}$, tension max. $10 \mathrm{~N}$ \\
& Presión estática (P) & $<10 \mathrm{MPa}$ \\
\hline
\end{tabular}

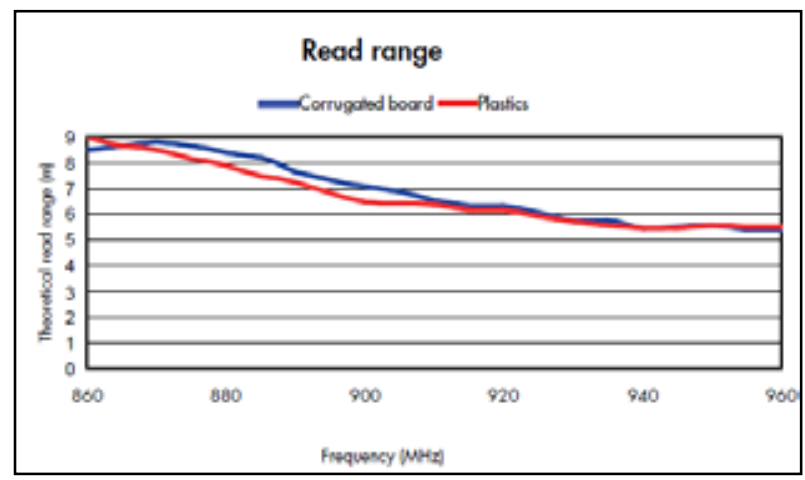

Antena Tipo Far Field - Antena con polarización circular tipo panel

\begin{tabular}{|lll|}
\hline & Rango de frecuencia & $902-928 \mathrm{MHz}$ \\
& Ganancia & $9 \mathrm{dBiC}$ \\
& VSWR Máximo & $1.3: 1$ \\
& $3 \mathrm{~dB}$ Beamwidth - Azimuth & $70^{\circ}$ \\
& Radio de alcance de frente al fondo & $20 \mathrm{~dB}$ \\
& Polarización & Circular Derecha o Izquierda \\
& Poder máximo de entrada & 10 Watts \\
& Impedancia de entrada & $50 \mathrm{hms}$ \\
& Axial ratio & $1 \mathrm{~dB}$ \\
& Peso (Kg) & 1.13 \\
& Dimensiones & $10.2^{\prime \prime} \times 10.2^{\prime \prime} \times 1.32^{\prime \prime}$ \\
& Conexión de la antena & Coax Pigtail, Rev TC Male \\
& Formas de adjuntarse & 4 Tornillos adheridos a la antena \\
& Temperatura adecuada de operación & $-25^{\circ} \mathrm{C}$ a $+70^{\circ} \mathrm{C}$ \\
& Protección eléctrica & Cables a tierra \\
IP54
\end{tabular}




\subsection{RESULTADOS}

Como ya se ha mencionado, en esta primera fase y a la fecha se ha concluido con el diseño de la planta del laboratorio RFID, la selección de los equipos más importantes para el inico de las actividades básicas, ejecución de obras complementarias de acondicionamiento para la instalación de los equipos y se está en pleno proceso de adquisición de los equipos seleccionados.

La idea de los participantes de esta investigación no es paralizar los trabajos a la entrega del presente informe técnico, sino continuar las actividades y programas en las tres fases de la investigación.

Con la aplicación de este proyecto se espera que esta tecnología novedosa en el Perú, el RFID, contribuya con la formación tanto en pregrado como en posgrado con nuevos conocimientos relacionados al tema, abriéndose así nuevos campos para la investigación incluyendo temas de tesis.

También es preciso indicar que una vez instalado los equipos del laboratorio RFID, se debe entrar a una etapa de ensayo con nuestros alumnos que han sido capacitados en el laboratorio de la empresa distribuidora de estos equipos, de tal forma que se desarrolle en las mismas instalaciones de la facultad una prueba piloto, así comprobaremos si es suficiente el tipo de instrumentos que se han adquirido o se necesita complementar con algunos otros más y verificar realmente si es ventajoso para una empresa implementar este tipo de tecnología en sus instalaciones, identificar las ventajas y elaborar el análisis de beneficio costo de la implementación para la toma de decisiones.

Los equipos seleccionados en la primera fase del proyecto son como sigue:

- Lectores RFID Impinj Revolution

- Elementos de corriente para reader

- Licencias de Software de control de dirección

- Convenios de garantías por 3 años

- Tags UPM Dog Bone Monza3

- Antenas Far field

\section{CONCLUSIONES}

1. La implementación del laboratorio de tecnología RFID ha demostrado ser exitosa en diferentes empresas del mundo, sin embargo, en el Perú son pocas las que la utilizan.

2. Con la implementación del laboratorio RFID la Facultad de Ingeniería Industrial y la UNMSM estarían a la vanguardia de tecnologías en este género, lo cual beneficiaría a sus alumnos tanto de pregrado como de posgrado, convirtiéndose en un centro de servicios y de capacitación también para los trabajadores de las empresas.

3. La Facultad de Ingeniería Industrial desarrollará una alianza estratégica con GS1 Perú para capacitar a los estudiantes y docentes en la tecnología RFID.

4. Se desarrollará una simulación para probar el modelo presentado.

5. Se incorporará al dictado de cursos tanto a docentes como a estudiantes del pregrado como del posgrado.

6. Este laboratorio formará parte del CEMA de la Facultad de Industriales, brindando así nuevos servicios, no solo a la facultad sino también como extensión social.

\section{REFERENCIAS BIBLIOGRÁFICAS}

[1] Ego México, “¿Qué es la identificación por radio frecuencia o RFID?", http://www.egomexico. com/tecnologia rfid.htm (4)

[2] EMPRESA GS1-Perú, Av. Javier Prado Oeste 2150, San Isidro, Lima-Perú (5)

[3] Gomis, Lluís Soler, entrevista 2006, director y fundador de IDtrack, http://www.rfid-magazine. com/entrevista/index.php?id=201

[4] Llamazares, Juan Carlos “¿Cómo funciona? Sistemas RFID”, http://www.ecojoven.com/ dos/03/RFID.html . (6)

[5] Noticiasdot.com, "Como funciona la tecnología para RFID”, http://www2.noticiasdot.com/publicaciones/2003/0403/0804/noticias080403/noticias080403-7-a.htm, 2003. (1)

[6] Perú Retail, http://noticiasretailperu.blogspot. com/2008/04/rfid-ms-all-de-unas-siglas.html (3)

[7] Sociedad de la información (ONTSI), Alberto Urueña (Coordinación). AETIC, Asociación de empresas de electrónica, tecnologías de la información, y telecomunicaciones de España; Antonio Cimorra, comisión RFID de AETIC, asistencia técnica: AT4 wireless S.A. Empresa tecnológica española-Málaga. Francisco Cañas, "La tecnología RFID: Usos y oportunidades". EDITA: Credes, Edificio Bronce, Plaza Manuel Gómez Moreno, s/n., 28020 Madrid. (7) 